\title{
PROPERTIES OF MEROMORPHIC SOLUTIONS OF SOME CERTAIN DIFFERENCE EQUATIONS
}

\author{
Chang-Wen Peng and Zong-Xuan Chen ${ }^{\dagger}$
}

\begin{abstract}
This paper considers some properties of meromorphic solutions of the nonlinear difference equation

$$
(f(z+1)+f(z))(f(z)+f(z-1))=\frac{P(z, f(z))}{Q(z, f(z))},
$$

where $P(z, f(z))$ and $Q(z, f(z))$ are polynomials in $f$ having rational coefficients and no common roots.
\end{abstract}

\section{Introduction and main results}

In what follows, we assume the reader is familiar with the basic notion of Nevanlinna's value distribution theory (see $[15,18,23])$. We also use notations $\sigma(f), \mu(f), \lambda(f), \lambda\left(\frac{1}{f}\right)$ for the order, the lower order, the exponents of convergence of zeros and poles of $f$, respectively. Moreover, we say that a meromorphic function $g$ is small with respect to $f$ if $T(r, g)=S(r, f)$, where $S(r, f)=o(T(r, f))$ outside a possible exceptional set of finite logarithmic measure. We also make use of the notion of hyper-order, defined as

$$
\sigma_{2}(f):=\varlimsup_{r \rightarrow \infty} \frac{\log \log T(r, f)}{\log r} .
$$

Recently, a number of papers (see $[1-8,10-14,16,17,19,21]$ ) focused on complex difference equations and difference analogues of Nevanlinna theory. As the difference analogues of Nevanlinna theory are being investigated, many results on the complex difference equations are rapidly obtained.

2010 Mathematics Subject Classification. primary 30D35, 39A10.

Key words and phrases. nonlinear difference equation; transcendental meromorphic solution; rational solution; hyper-order.

This project is supported by the National Natural Science Foundation of China (No: 11171119).

$\dagger$ Corresponding author.

Received January 22, 2013; revised July 26, 2013. 
Ablowitz, Halburd and Herbst [1] considered the difference equations of the type

$$
f(z+1) * f(z-1)=R(z, f(z)),
$$

where $R(z, f(z))$ is rational in both of its arguments, and the operation $*$ stands either for the addition or the multiplication. Ablowitz et.al. obtained the following theorem therein:

THEOREM A ([1]). If the second-order difference equation (1.1) admits a nonrational meromorphic solution of finite order, then $\operatorname{deg}_{f}(R) \leq 2$.

The following theorem is due to Ramani, Grammaticos, Tamizhmani and Tamizhmani.

THEOREM B ([20]). If the second-order difference equation

$$
(f(z+1)+f(z))(f(z)+f(z-1))=\frac{P(z, f(z))}{Q(z, f(z))},
$$

where $P(z, f(z))$ and $Q(z, f(z))$ are polynomials in $f$ having rational coefficients and no common roots, admits a non-rational meromorphic solution of finite order, then $\operatorname{deg}_{f}(P) \leq 4$ and $\operatorname{deg}_{f}(Q) \leq 2$.

In Theorem B, we see that if equation (1.2) admits a transcendental meromorphic solution of finite order, then $\operatorname{deg}_{f}(P) \leq 4$ and $\operatorname{deg}_{f}(Q) \leq 2$. It is natural to ask if $p=\operatorname{deg}_{f}(P) \geq 4$ and $q=\operatorname{deg}_{f}(Q) \geq 2$ or $p-q \geq 3$, what do we get?

In the following, we will answer the above questions, and obtain Theorem 1.1 as show below.

THEOREM 1.1. Suppose that $f$ is a transcendental meromorphic solution of the equation

$$
(f(z+1)+f(z))(f(z)+f(z-1))=R(z, f(z))=\frac{P(z, f(z))}{Q(z, f(z))},
$$

where $P(z, f(z))=a_{0}(z)+a_{1}(z) f(z)+\cdots+a_{p}(z) f(z)^{p}$ and $Q(z, f(z))=b_{0}(z)+$ $b_{1}(z) f(z)+\cdots+b_{q}(z) f(z)^{q}$ are relatively prime polynomials in $f, a_{0}(z), \ldots, a_{p}(z)$, $b_{0}(z), \ldots, b_{q}(z)$ are rational functions with $a_{p}(z) b_{q}(z) \not \equiv 0$. Let $m=p-q \geq 3$.

1) If $f$ is entire or has finitely many poles, then there exist constants $K>0$ and $r_{0}>0$ such that

$$
\log M(r, f) \geq K\left(\frac{m}{2}\right)^{r}
$$

holds for all $r \geq r_{0}$. 
2) If $f$ has infinitely many poles, then there exist constants $K>0$ and $r_{0}>0$ such that

$$
n(r, f) \geq K(m-1)^{r}
$$

holds for all $r \geq r_{0}$.

Furthermore, by 1) and 2), we can get $\sigma_{2}(f) \geq 1$.

From Theorem B and Theorem 1.1, we can get the following Corollary 1.1 .

COROLlaRY 1.1. Suppose that the second-order difference equation (1.2) satisfies the hypothesis of Theorem $B$. If equation (1.2) admits a non-rational meromorphic solution of finite order, then $\operatorname{deg}_{f}(P) \leq 4, \quad \operatorname{deg}_{f}(Q) \leq 2$ and $\operatorname{deg}_{f}(P)-\operatorname{deg}_{f}(Q) \leq 2$.

Remark 1.1. Under the conditions of $\operatorname{deg}_{f}(P) \leq 4, \operatorname{deg}_{f}(Q) \leq 2$ and $\operatorname{deg}_{f}(P)-\operatorname{deg}_{f}(Q) \leq 2$, equation (1.2) may have meromorphic solution of infinite order, which can be seen by the following example.

Example 1.1. The difference equation

$$
(f(z+1)+f(z))(f(z)+f(z-1))=4 f^{2}(z)
$$

has a solution $f(z)=\exp \left\{e^{2 \pi i z}\right\}$, where $\sigma(f)=\infty$.

In what follows, we consider the properties of meromorphic solutions of finite order of equation (1.3), and get Theorem 1.2.

THEOREM 1.2. Suppose that equation (1.3) satisfies the hypothesis of Theorem 1.1 , and $f$ is a finite order transcendental meromorphic solution of equation (1.3). Then, we have

1) If $p=q=0$, then $\sigma(f) \geq 1$.

2) If $a_{0}(z) \not \equiv 0$, then $\lambda(f)=\sigma(f)$.
3) If $q \geq p, q \geq 1$ and $P(z, f(z)) \not \equiv 0$, then $\lambda\left(\frac{1}{f}\right)=\sigma(f)$. 4) If $P(z, f(z)) \neq \equiv 0$, then $\max \left\{\lambda(f), \lambda\left(\frac{1}{f}\right)\right\}=\sigma(f)$ except $(f(z+1)+$
$f(z))(f(z)+f(z-1))=a_{2}(z) f^{2}(z)$.

Remark 1.2. If $P(z, f(z)) \equiv 0$, we may get $\lambda\left(\frac{1}{f}\right)<\sigma(f)$. And when $P(z, f(z))=a_{2}(z) f^{2}(z)$, we may get $\max \left\{\lambda(f), \lambda\left(\frac{1}{f}\right)\right\}<\sigma(f)$. Which can be
seen by the following two examples. 
Example 1.2. The difference equation

$$
(f(z+1)+f(z))(f(z)+f(z-1))=0
$$

has a solution $f(z)=e^{\pi i z}$, where $\lambda\left(\frac{1}{f}\right)=0$ and $\sigma(f)=1$.

Example 1.3. The difference equation

$$
(f(z+1)+f(z))(f(z)+f(z-1))=4 f^{2}(z)
$$

has a solution $f(z)=e^{2 \pi i z}$, where $\max \left\{\lambda(f), \lambda\left(\frac{1}{f}\right)\right\}=0$ and $\sigma(f)=1$.

When $\operatorname{deg}_{f}(P)=0$ and $\operatorname{deg}_{f}(Q)=0$, we consider the properties of rational solutions of equation (1.3), and get Theorem 1.3. THEOREM 1.3. Let $R(z)=\frac{P(z)}{Q(z)}$ be an irreducible rational function. We
consider the difference equation

$$
(f(z+1)+f(z))(f(z)+f(z-1))=\frac{P(z)}{Q(z)},
$$

where $P(z), Q(z)$ are polynomials with $\operatorname{deg} P(z)=p$ and $\operatorname{deg} Q(z)=q$.

1) Suppose that $p \geq q$ and $p-q$ is an even number or zero. If equation (1.6) has an irreducible rational solution $f(z)=\frac{m(z)}{n(z)}$, where $m(z), n(z)$ are polynomials with $\operatorname{deg} m(z)=m$ and $\operatorname{deg} n(z)=n$, then

$$
m-n=\frac{p-q}{2} .
$$

2) Suppose that $p<q$ and $q-p$ is an even number. If equation (1.6) has an irreducible rational solution $f(z)=\frac{m(z)}{n(z)}$, where $m(z), n(z)$ are polynomials with $\operatorname{deg} m(z)=m$ and $\operatorname{deg} n(z)=n$, then

$$
n-m=\frac{q-p}{2} .
$$

3) If $|p-q|$ is an odd number, then equation (1.6) has no rational solution.

\section{Proof of Theorem 1.1}

1) We multiply out the denominators of the coefficients $a_{i}(z)(i=0$, $1, \ldots, p), b_{n}(z)(n=0,1, \ldots, q)$ in $(1.3)$ to obtain

$$
(f(z+1)+f(z))(f(z)+f(z-1))=R_{1}(z, f(z)),
$$


where $R_{1}(z, f(z))=\frac{A_{0}(z)+A_{1}(z) f(z)+\cdots+A_{p}(z) f(z)^{p}}{B_{0}(z)+B_{1}(z) f(z)+\cdots+B_{q}(z) f(z)^{q}}$, and all coefficients $A_{i}(z)(i=0,1, \ldots, p), B_{n}(z)(n=0,1, \ldots, q)$ are polynomials.

Suppose that $f$, the solution of (2.1), is transcendental entire. Furthermore, denote $l_{n}=\operatorname{deg} B_{n}, t=\operatorname{deg} A_{p}$. The maximum modulus principle yields

$$
M(r+1, f(z)) \geq M(r, f(z \pm 1))
$$

for $z$ satisfying $|z|=r$. Choosing $d>\max \left\{l_{0}, l_{1}, \ldots, l_{q}\right\}$, it follows that

$$
\begin{aligned}
M\left(r, R_{1}(z, f(z))\right) & =M(r,(f(z+1)+f(z))(f(z)+f(z-1))) \\
& \leq M\left(r+1,4 f(z)^{2}\right) \leq C M^{2}(r+1, f(z)),
\end{aligned}
$$

when $r$ is large enough, where $C$ is a positive constant. Furthermore

$$
\begin{aligned}
\left|\sum_{i=0}^{p} A_{i}(z) f(z)^{i}\right| & \geq\left|A_{p}(z) f(z)^{p}\right|-\left(\left|A_{p-1}(z) f(z)^{p-1}\right|+\cdots+\left|A_{0}(z)\right|\right) \\
& \geq \frac{1}{2}\left|A_{p}(z) f(z)^{p}\right|=\frac{1}{2} r^{t}|f(z)|^{p}(1+o(1))
\end{aligned}
$$

for $z$ satisfying $|z|=r$ such that $|f(z)|=M(r, f)$. And

$$
\begin{aligned}
\left|\sum_{n=0}^{q} B_{n}(z) f(z)^{n}\right| & \leq \sum_{n=0}^{q}\left|B_{n}(z) f(z)^{n}\right| \\
& \leq \sum_{n=0}^{q} r^{d}|f(z)|^{q}=(q+1) r^{d}|f(z)|^{q},
\end{aligned}
$$

when $r$ is large enough. Hence

$$
\begin{aligned}
\left|R_{1}(z, f(z))\right| & =\left|\frac{\sum_{i=0}^{p} A_{i}(z) f(z)^{i}}{\sum_{n=0}^{q} B_{n}(z) f(z)^{n}}\right| \\
& \geq \frac{\left|A_{p}(z) f(z)^{p}\right|-\left(\left|A_{p-1}(z) f(z)^{p-1}\right|+\cdots+\left|A_{0}(z)\right|\right)}{\left|B_{q}(z) f(z)^{q}\right|+\cdots+\left|B_{1}(z) f(z)\right|+\left|B_{0}(z)\right|} \\
& \geq \frac{\frac{1}{2} r^{t}|f(z)|^{p}(1+o(1))}{(q+1) r^{d}|f(z)|^{q}}=\frac{1}{2(q+1)} r^{(t-d)}|f(z)|^{(p-q)}(1+o(1)),
\end{aligned}
$$

when $r$ is sufficiently large and for $z$ satisfying $|z|=r$ such that $|f(z)|=M(r, f)$. So that we have

$$
M\left(r, R_{1}(z, f(z))\right) \geq \frac{r^{(t-d)}|f(z)|^{p-q}}{2(q+1)}=\frac{r^{(t-d)} M(r, f(z))^{m}}{2(q+1)},
$$


when $r$ is sufficiently large and for $z$ satisfying $|z|=r$ such that $|f(z)|=M(r, f)$. By (2.2) and (2.3), we deduce

$$
2 \log M(r+1, f(z)) \geq m \log M(r, f(z))+g(r),
$$

where $|g(r)|<K \log r$ for some $K>0$, when $r$ is large enough. By iterating (2.4), we have that

$$
\log M(r+j, f(z)) \geq\left(\frac{m}{2}\right)^{j} \log M(r, f(z))+E_{j}(r),
$$

where

$$
\begin{aligned}
\left|E_{j}(r)\right| & =\frac{1}{2}\left|\left(\frac{m}{2}\right)^{j-1} g(r)+\left(\frac{m}{2}\right)^{j-2} g(r+1)+\cdots+g(r+(j-1))\right| \\
& \leq \frac{K}{2}\left(\frac{m}{2}\right)^{j-1} \sum_{k=0}^{j-1} \frac{\log (r+k)}{\left(\frac{m}{2}\right)^{k}} \leq \frac{K}{2}\left(\frac{m}{2}\right)^{j-1} \sum_{k=0}^{\infty} \frac{\log (r+k)}{\left(\frac{m}{2}\right)^{k}} .
\end{aligned}
$$

Since $\log (r+k) \leq(\log r)(\log k)$ for $r$ and $k$ sufficiently large, we have that

$$
\sum_{k=0}^{\infty} \frac{\log (r+k)}{\left(\frac{m}{2}\right)^{k}} \leq \sum_{k=0}^{\infty} \frac{(\log r)(\log k)}{\left(\frac{m}{2}\right)^{k}}=\log r \sum_{k=0}^{\infty} \frac{\log k}{\left(\frac{m}{2}\right)^{k}}
$$

Set

$$
I=\sum_{k=0}^{\infty} a_{k}=\sum_{k=0}^{\infty} \frac{\log k}{\left(\frac{m}{2}\right)^{k}}
$$

Since

$$
\lim _{k \rightarrow \infty} \frac{a_{k+1}}{a_{k}}=\lim _{k \rightarrow \infty} \frac{\log (k+1) /\left(\frac{m}{2}\right)^{k+1}}{\log k /\left(\frac{m}{2}\right)^{k}}=\lim _{k \rightarrow \infty} \frac{\log (k+1)}{\log k} \frac{2}{m}=\frac{2}{m}<1,
$$

we see that the series $I=\sum_{k=0}^{\infty} \frac{\log k}{\left(\frac{m}{2}\right)^{k}}$ is convergent. Hence

$$
\left|E_{j}(r)\right| \leq K^{\prime}\left(\frac{m}{2}\right)^{j} \log r .
$$


Since, by the hypothesis, $f$ is transcendental entire, we have the inequality $\log M(r, f) \geq 2 K^{\prime} \log r$ for $r$ large enough. Thus (2.5) and (2.6) imply

$$
\log M(r+j, f(z)) \geq K^{\prime}\left(\frac{m}{2}\right)^{j} \log r
$$

which holds for $r$ sufficiently large, say $r \geq r_{0}$. By choosing $r \in\left[r_{0}, r_{0}+1\right)$ arbitrarily and letting $j \rightarrow \infty$ for each choice of $r$, and set $s=r+j$, then $j=s-r \geq s-\left(r_{0}+1\right)$. Thus, we have

$$
\log M(s, f(z))=\log M(r+j, f(z)) \geq K^{\prime}\left(\frac{m}{2}\right)^{s-r_{0}-1} \log r_{0}=K^{\prime \prime}\left(\frac{m}{2}\right)^{s}
$$
holds for all $s \geq s_{0}=r_{0}+1$, where $K^{\prime \prime}=K^{\prime}\left(\frac{m}{2}\right)^{-\left(r_{0}+1\right)} \log r_{0}$. We have proved
the assertion in the case of $f$ being entire.

Suppose now that $f$, the solution of (1.3), is meromorphic with finitely many poles. Then there exists a polynomial $P(z)$ such that $g(z)=P(z) f(z)$ is entire. Substituting $f(z)=\frac{g(z)}{P(z)}$ into (1.3) and again multiplying away the denominators, we will obtain an equation similar to (2.1). Applying the same reasoning above to $g(z)$, we obtain that for sufficiently large $r, \log M(r, f)=\log M(r, g)+$ $O(\log r) \geq\left(K^{\prime \prime}-\varepsilon\right)\left(\frac{m}{2}\right)^{r}=K^{\prime \prime \prime}\left(\frac{m}{2}\right)^{r}$, where $K^{\prime \prime \prime}(>0)$ is some constant.

2) We multiply out the denominators of the coefficients $a_{i}(z)(i=0$, $1, \ldots, p), b_{n}(z)(n=0,1, \ldots, q)$ in (1.3) to obtain (2.1). Suppose that $f(z)$ is a meromorphic function with infinitely many poles. Since $A_{i}(z)(i=0,1, \ldots, p)$, $B_{n}(z)(n=0,1, \ldots, q)$ are polynomials, we see that there is a constant $M>0$ such that all zeros of $A_{i}(z)(i=0,1, \ldots, p), B_{n}(z)(n=0,1, \ldots, q)$ are in $D=$ $\{z:|\operatorname{Re} z|<M,|\operatorname{Im} z|<M\}$.

Set

$$
\begin{array}{ll}
D_{1}=\{z: \operatorname{Re} z>M\} ; & D_{2}=\{z: \operatorname{Re} z<-M\} ; \\
D_{3}=\{z: \operatorname{Im} z>M\} ; & D_{4}=\{z: \operatorname{Im} z<-M\} .
\end{array}
$$

Since $f(z)$ has infinitely many poles, we see that there exists at least one of $D_{s}(s=1,2,3,4)$ such that $f(z)$ has infinitely many poles in it. Suppose that $z_{0}$ is in one of $D_{s}(s=1,2,3,4)$ such that $D_{s}$ has infinitely many poles of $f(z)$, and $z_{0}$ is a pole of $f(z)$ having multiplicity $k_{0} \geq 1$. Then the right-hand side of $(2.1)$ has a pole of multiplicity $m k_{0}$ at $z_{0}$. Thus, there is $l_{1} \in\{1,-1\}$ such that $z_{0}+l_{1}$ is a pole of $f(z)$ of multiplicity $k_{1} \geq \frac{m k_{0}}{2}$.

We divide this proof into the following two cases. $k_{1}$.

CASE 1. Suppose that $l_{1}=1$. Then $z_{0}+1$ is a pole of $f(z)$ of multiplicity 
Suppose that $f(z)$ has infinitely many poles in $D_{1}$ and $z_{0} \in D_{1}$. Then $z_{0}+1 \in D_{1}$ since $z_{0} \in D_{1}$. Substitute $z_{0}+1$ for $z$ in (2.1) to obtain

$$
\begin{aligned}
& \left(f\left(z_{0}+2\right)+f\left(z_{0}+1\right)\right)\left(f\left(z_{0}+1\right)+f\left(z_{0}\right)\right) \\
& \quad=\frac{A_{0}\left(z_{0}+1\right)+\cdots+A_{p}\left(z_{0}+1\right) f^{p}\left(z_{0}+1\right)}{B_{0}\left(z_{0}+1\right)+\cdots+B_{q}\left(z_{0}+1\right) f^{q}\left(z_{0}+1\right)} .
\end{aligned}
$$

That is

$$
\begin{aligned}
f\left(z_{0}\right. & +2) f\left(z_{0}+1\right)+f\left(z_{0}+2\right) f\left(z_{0}\right)+f^{2}\left(z_{0}+1\right)+f\left(z_{0}+1\right) f\left(z_{0}\right) \\
& =\frac{A_{0}\left(z_{0}+1\right)+\cdots+A_{p}\left(z_{0}+1\right) f^{p}\left(z_{0}+1\right)}{B_{0}\left(z_{0}+1\right)+\cdots+B_{q}\left(z_{0}+1\right) f^{q}\left(z_{0}+1\right)} .
\end{aligned}
$$

By (2.8) and $m=p-q \geq 3$, we conclude that $z_{0}+2$ is a pole of $f(z)$ of multiplicity $k_{2}=(m-1) k_{1}$. Obviously $z_{0}+2 \in D_{1}$.

Substitute $z_{0}+2$ for $z$ in (2.1) to obtain

$$
\begin{aligned}
f\left(z_{0}+3\right) f\left(z_{0}+2\right)+f\left(z_{0}+3\right) f\left(z_{0}+1\right)+f^{2}\left(z_{0}+2\right)+f\left(z_{0}+2\right) f\left(z_{0}+1\right) \\
\quad=\frac{A_{0}\left(z_{0}+2\right)+\cdots+A_{p}\left(z_{0}+2\right) f^{p}\left(z_{0}+2\right)}{B_{0}\left(z_{0}+2\right)+\cdots+B_{q}\left(z_{0}+2\right) f^{q}\left(z_{0}+2\right)} .
\end{aligned}
$$

By (2.9) and $m=p-q \geq 3$, we conclude that $z_{0}+3$ is a pole of $f(z)$ of multiplicity $k_{3}=(m-1) k_{2}=(m-1)^{2} k_{1}$. Obviously $z_{0}+3 \in D_{1}$.

Similarly, $z_{0}+n \in D_{1}$ is a pole of $f(z)$ of multiplicity $k_{n}=(m-1) k_{n-1}$ $=\cdots=(m-1)^{n-1} k_{1}$. Thus, there is a sequence $\left\{z_{0}+j \in D_{1}(j=1,2, \ldots\right.$, $n, \ldots)\}$ are poles of $f(z)$ of multiplicity $k_{j}=(m-1)^{j-1} k_{1}$. Since $k_{j}=$ $(m-1)^{j-1} k_{1} \rightarrow \infty$, as $j \rightarrow \infty$, and since $f(z)$ does not have essential singularities in the finite plane, we must have $\left|z_{0}+j\right| \rightarrow \infty$, as $j \rightarrow \infty$. It is clear that, for $j$ large enough, say $j>j_{0}$,

$$
\begin{aligned}
(m-1)^{j-1} k_{1} & \leq k_{1}\left(1+(m-1)+\cdots+(m-1)^{j-1}\right) \\
& \leq n\left(\left|z_{0}+j\right|, f\right) \leq n\left(\left|z_{0}\right|+j, f\right) \leq n(t+j, f),
\end{aligned}
$$

where $t \in\left[\left|z_{0}\right|,\left|z_{0}\right|+1\right]$ can be chosen arbitrarily. Letting $j \rightarrow \infty$ for each choice of $t$, and set $r=t+j$, then $j-1=r-t-1 \geq r-\left(\left|z_{0}\right|+2\right)$. Thus, we have

$$
n(r, f) \geq(m-1)^{j-1} k_{1} \geq k_{1}(m-1)^{r-\left(\left|z_{0}\right|+2\right)}=K(m-1)^{r}
$$

holds for all $r \geq r_{0}:=j_{0}+1+\left|z_{0}\right|$, where $K=k_{1}(m-1)^{-\left(\left|z_{0}\right|+2\right)}$. The fact that $r_{0}$ and $K$ both depend on $\left|z_{0}\right|$ is not a problem, since $z_{0}$ is fixed.

Suppose that $f(z)$ has infinitely many poles in $D_{3}$ (or $D_{4}$ ). Then we may use the same method as above.

Suppose that $f(z)$ has infinitely many poles in $D_{2}$ and $z_{0} \in D_{2}$. Set $\operatorname{deg} A_{p}=A(\geq 0)$. Since $z_{0} \in D_{2}$, we know that $z_{0}+1$ has two possibilities:

(i) If $z_{0}+1 \notin D_{2}$, this process will be terminated and we have to choose another pole $z_{0}$ of $f(z)$ in the way we did above. 
(ii) If $z_{0}+1 \in D_{2}$, then $z_{0}+1$ is a pole of $f(z)$ of multiplicity $k_{1} \geq \frac{m k_{0}}{2}$, since the right-hand side of $(2.1)$ has a pole of multiplicity $m k_{0}$ at $z_{0}$.

Substitute $z_{0}+1$ for $z$ in (2.1) to obtain (2.8). And we conclude that $z_{0}+2$ is a pole of $f(z)$ of multiplicity $k_{2}=(m-1) k_{1}$.

If $z_{0}+2 \notin D_{2}$, this process will be terminated and we have to choose another pole $z_{0}$ of $f(z)$ in the way we did above.

If $z_{0}+2 \in D_{2}$, then we see that the right-hand side of (2.1) has a pole of multiplicity $m k_{2}$ at $z_{0}+2$.

Substitute $z_{0}+2$ for $z$ in (2.1) to obtain (2.9). Hence we see that $z_{0}+3$ is a pole of $f(z)$ of multiplicity $k_{3}=(m-1) k_{2}=(m-1)^{2} k_{1}$.

We proceed to follow the steps (i) and (ii) as above. Since there are infinitely many poles of $f(z)$ in $D_{2}$, we will find a pole $z_{0}\left(\in D_{2}\right)$ of $f(z)$ such that $z_{0}+n_{1}\left(\in D_{2}\right)$ is a pole of $f(z)$ of multiplicity $k_{n_{1}}=(m-1) k_{\left(n_{1}-1\right)}=\cdots=$ $(m-1)^{n_{1}-1} k_{1}$. And $z_{0}$ satisfies $z_{0}+n_{1}+1 \notin D_{2}$, that is $\operatorname{Re}\left(z_{0}+n_{1}+1\right) \geq-M$. By (2.1) and $m=p-q \geq 3$, we conclude that $z_{0}+n_{1}+1$ is a pole of $f(z)$ of multiplicity $k_{\left(n_{1}+1\right)}=(m-1) k_{n_{1}}=(m-1)^{n_{1}} k_{1}$.

Substitute $z_{0}+n_{1}+1$ for $z$ in (2.1) to obtain

$$
\begin{aligned}
f\left(z_{0}+\right. & \left.n_{1}+2\right) f\left(z_{0}+n_{1}+1\right)+f\left(z_{0}+n_{1}+2\right) f\left(z_{0}+n_{1}\right) \\
& +f^{2}\left(z_{0}+n_{1}+1\right)+f\left(z_{0}+n_{1}+1\right) f\left(z_{0}+n_{1}\right) \\
= & \frac{A_{0}\left(z_{0}+n_{1}+1\right)+\cdots+A_{p}\left(z_{0}+n_{1}+1\right) f^{p}\left(z_{0}+n_{1}+1\right)}{B_{0}\left(z_{0}+n_{1}+1\right)+\cdots+B_{q}\left(z_{0}+n_{1}+1\right) f^{q}\left(z_{0}+n_{1}+1\right)} .
\end{aligned}
$$

We see that the right-hand side of $(2.10)$ has a pole of multiplicity at least $p k_{\left(n_{1}+1\right)}-A-q k_{\left(n_{1}+1\right)}=m k_{\left(n_{1}+1\right)}-A$ at $z_{0}+n_{1}+1$. Without loss of generality, suppose that the right-hand side of $(2.10)$ has a pole of multiplicity $m k_{\left(n_{1}+1\right)}-A$ at $z_{0}+n_{1}+1$.

In the left-hand side of (2.10), we see that $f^{2}(z)$ has a pole of multiplicity $2 k_{\left(n_{1}+1\right)}$ at $z_{0}+n_{1}+1, f(z) f(z-1)$ has a pole of multiplicity $k_{n_{1}}+k_{\left(n_{1}+1\right)}$ at $z_{0}+n_{1}+1$. By $m \geq 3$, when $n_{1}>\max \left\{\frac{\log (A+1)-\log (m-2) k_{1}}{\log (m-1)}, 1\right\}$, we have $(m-2) k_{\left(n_{1}+1\right)}=(m-2)(m-1)^{n_{1}} k_{1}>A+1>A$. Thus $m k_{\left(n_{1}+1\right)}-A>2 k_{\left(n_{1}+1\right)}$ and $2 k_{\left(n_{1}+1\right)}>k_{n_{1}}+k_{\left(n_{1}+1\right)}$.

Hence, by (2.10), we conclude that $z_{0}+n_{1}+2$ is a pole of $f(z)$ of multiplicity $k_{\left(n_{1}+2\right)}=m k_{\left(n_{1}+1\right)}-A-k_{\left(n_{1}+1\right)}=(m-1) k_{\left(n_{1}+1\right)}-A=(m-1)^{n_{1}+1} k_{1}-A$.

Since $\operatorname{Re}\left(z_{0}+n_{1}+1\right) \geq-M$, we have $\operatorname{Re}\left(z_{0}+n_{1}+2\right)>\operatorname{Re}\left(z_{0}+n_{1}+1\right) \geq$ $-M$. Substitute $z_{0}+n_{1}+2$ for $z$ in (2.1) to obtain

$$
\begin{aligned}
f\left(z_{0}+\right. & \left.n_{1}+3\right) f\left(z_{0}+n_{1}+2\right)+f\left(z_{0}+n_{1}+3\right) f\left(z_{0}+n_{1}+1\right) \\
& +f^{2}\left(z_{0}+n_{1}+2\right)+f\left(z_{0}+n_{1}+2\right) f\left(z_{0}+n_{1}+1\right) \\
= & \frac{A_{0}\left(z_{0}+n_{1}+2\right)+\cdots+A_{p}\left(z_{0}+n_{1}+2\right) f^{p}\left(z_{0}+n_{1}+2\right)}{B_{0}\left(z_{0}+n_{1}+2\right)+\cdots+B_{q}\left(z_{0}+n_{1}+2\right) f^{q}\left(z_{0}+n_{1}+2\right)} .
\end{aligned}
$$


We see that the right-hand side of (2.11) has a pole of multiplicity at least $p k_{\left(n_{1}+2\right)}-A-q k_{\left(n_{1}+2\right)}=m k_{\left(n_{1}+2\right)}-A$ at $z_{0}+n_{1}+2$. Without loss of generality, suppose that the right-hand side of (2.11) has a pole of multiplicity $m k_{\left(n_{1}+2\right)}-A$ at $z_{0}+n_{1}+2$.

In the left-hand side of (2.11), we see that $f^{2}(z)$ has a pole of multiplicity $2 k_{\left(n_{1}+2\right)}$ at $z_{0}+n_{1}+2, f(z) f(z-1)$ has a pole of multiplicity $k_{\left(n_{1}+1\right)}+k_{\left(n_{1}+2\right)}$ at $z_{0}+n_{1}+2$. By $m \geq 3$, when $n_{1}>\max \left\{\frac{\log (A+1)-\log (m-2) k_{1}}{\log (m-1)}, 1\right\}$, we have $(m-2)(m-1)^{n_{1}} k_{1}>A+1>A$. Hence, $\quad(m-2) k_{\left(n_{1}+2\right)}=$ $(m-2)\left[(m-1)^{\left(n_{1}+1\right)} k_{1}-A\right]>A$. Thus $m k_{\left(n_{1}+2\right)}-A>2 k_{\left(n_{1}+2\right)}$ and $2 k_{\left(n_{1}+2\right)}>$ $k_{\left(n_{1}+1\right)}+k_{\left(n_{1}+2\right)}$.

Hence, by (2.11), we conclude that $z_{0}+n_{1}+3$ is a pole of $f(z)$ of multiplicity $k_{\left(n_{1}+3\right)}=m k_{\left(n_{1}+2\right)}-A-k_{\left(n_{1}+2\right)}=(m-1) k_{\left(n_{1}+2\right)}-A=(m-1)\left[(m-1)^{n_{1}+1} k_{1}-A\right]$ $-A=(m-1)^{n_{1}+2} k_{1}-A[(m-1)+1]$.

We proceed to follow the steps as above. We will find $z_{0}+n_{1}+n_{2}$ is a pole of $f(z)$ of multiplicity $k_{\left(n_{1}+n_{2}\right)}=(m-1)^{n_{1}+n_{2}-1} k_{1}-A\left[(m-1)^{n_{2}-2}+\cdots+\right.$ $(m-1)+1]$ such that $\operatorname{Re}\left(z_{0}+n_{1}+n_{2}\right)>M$, that is $z_{0}+n_{1}+n_{2} \in D_{1}$.

Then

Set $\quad k:=k_{\left(n_{1}+n_{2}\right)}=(m-1)^{n_{1}+n_{2}-1} k_{1}-A\left[(m-1)^{n_{2}-2}+\cdots+(m-1)+1\right]$.

$$
k=(m-1)^{n_{1}+n_{2}-1} k_{1}-A \frac{(m-1)^{n_{2}-1}-1}{(m-1)-1} .
$$

That is

$$
\begin{aligned}
k & =(m-1)^{n_{1}+n_{2}-1} k_{1}-A \frac{(m-1)^{n_{2}-1}}{m-2}+\frac{A}{m-2} \\
& =\frac{(m-1)^{n_{2}-1}}{m-2}\left[(m-2)(m-1)^{n_{1}} k_{1}-A\right]+\frac{A}{m-2} .
\end{aligned}
$$

When $\quad n_{2} \geq 2 \quad$ and $\quad n_{1}>\max \left\{\frac{\log (A+1)-\log (m-2) k_{1}}{\log (m-1)}, 1\right\}, \quad$ we have $(m-2)(m-1)^{n_{1}} k_{1}>A+1$, that is $(m-2)(m-1)^{n_{1}} k_{1}-A>1$. Hence we see that

$$
k=\frac{(m-1)^{n_{2}-1}}{m-2}\left[(m-2)(m-1)^{n_{1}} k_{1}-A\right]+\frac{A}{m-2} \geq 1 .
$$

Set $z_{1}:=z_{0}+n_{1}+n_{2}$. Then $z_{1}\left(\in D_{1}\right)$ is a pole of $f(z)$ of multiplicity $k \geq 1$.

Applying the same reasoning that $f(z)$ has infinitely many poles in $D_{1}$, we obtain

$$
n(r, f) \geq K(m-1)^{r}
$$

holds for all $r \geq r_{0}$. The fact that $r_{0}$ and $K$ both depend on $\left|z_{1}\right|$ is not a problem, since $z_{1}\left(\in D_{1}\right)$ is fixed by $z_{0}\left(\in D_{2}\right)$. 
CASE 2. Suppose that $l_{1}=-1$. Then $z_{0}-1$ is a pole of $f(z)$ of multiplicity $k_{1} \geq \frac{m k_{0}}{2}$.

Suppose that $f(z)$ has infinitely many poles in $D_{2}$ and $z_{0} \in D_{2}$. Then $z_{0}-1 \in D_{2}$ since $z_{0} \in D_{2}$. Substitute $z_{0}-1$ for $z$ in (2.1) to obtain

$$
\begin{gathered}
f\left(z_{0}\right) f\left(z_{0}-1\right)+f\left(z_{0}\right) f\left(z_{0}-2\right)+f^{2}\left(z_{0}-1\right)+f\left(z_{0}-1\right) f\left(z_{0}-2\right) \\
=\frac{A_{0}\left(z_{0}-1\right)+\cdots+A_{p}\left(z_{0}-1\right) f^{p}\left(z_{0}-1\right)}{B_{0}\left(z_{0}-1\right)+\cdots+B_{q}\left(z_{0}-1\right) f^{q}\left(z_{0}-1\right)} .
\end{gathered}
$$

By (2.12) and $m=p-q \geq 3$, we conclude that $z_{0}-2$ is a pole of $f(z)$ of multiplicity $k_{2}=(m-1) k_{1}$. Obviously $z_{0}-2 \in D_{2}$.

Similarly, $z_{0}-n\left(\in D_{2}\right)$ is a pole of $f(z)$ of multiplicity $k_{n}=(m-1)^{n-1} k_{1}$. Thus, there is a sequence $\left\{z_{0}-j \in D_{2}(j=1,2, \ldots, n, \ldots)\right\}$ are poles of $f(z)$ of multiplicity $k_{j}=(m-1)^{j-1} k_{1}$. Since $k_{j}=(m-1)^{j-1} k_{1} \rightarrow \infty$, as $j \rightarrow \infty$, and since $f$ does not have essential singularities in the finite plane, we must have $\left|z_{0}-j\right| \rightarrow \infty$, as $j \rightarrow \infty$. It is clear that, for $j$ large enough, say $j>j_{0}$,

$$
\begin{aligned}
(m-1)^{j-1} k_{1} & \leq k_{1}\left(1+(m-1)+\cdots+(m-1)^{j-1}\right) \\
& \leq n\left(\left|z_{0}-j\right|, f\right) \leq n\left(\left|z_{0}\right|+j, f\right) \leq n(t+j, f),
\end{aligned}
$$

where $t \in\left[\left|z_{0}\right|,\left|z_{0}\right|+1\right]$ can be chosen arbitrarily. Letting $j \rightarrow \infty$ for each choice of $t$, and set $r=t+j$, then $j-1=r-t-1 \geq r-\left(\left|z_{0}\right|+2\right)$. Thus, we have that

$$
n(r, f) \geq(m-1)^{j-1} k_{1} \geq k_{1}(m-1)^{r-\left(\left|z_{0}\right|+2\right)}=K(m-1)^{r}
$$

holds for all $r \geq r_{0}:=j_{0}+1+\left|z_{0}\right|$, where $K=k_{1}(m-1)^{-\left(\left|z_{0}\right|+2\right)}$. The fact that $r_{0}$ and $K$ both depend on $\left|z_{0}\right|$ is not a problem, since $z_{0}$ is fixed.

Suppose that $f(z)$ has infinitely many poles in $D_{3}$ (or $D_{4}$ ). Then we may use the same method as above.

Suppose that $f(z)$ has infinitely many poles in $D_{1}$ and $z_{0} \in D_{1}$. Set $\operatorname{deg} A_{p}=A \geq 0$. Since $z_{0} \in D_{1}$, we know that $z_{0}-1$ has two possibilities:

(i) If $z_{0}-1 \notin D_{1}$, this process will be terminated and we have to choose another pole $z_{0}$ of $f(z)$ in the way we did above.

(ii) If $z_{0}-1 \in D_{1}$, then $z_{0}-1$ is a pole of $f(z)$ of multiplicity $k_{1} \geq \frac{m k_{0}}{2}$, since the right-hand side of $(2.1)$ has a pole of multiplicity $m k_{0}$ at $z_{0}$.

Substitute $z_{0}-1$ for $z$ in (2.1) to obtain (2.12). And we conclude that $z_{0}-2$ is a pole of $f(z)$ of multiplicity $k_{2}=(m-1) k_{1}$.

We proceed to follow the steps (i) and (ii) as above. Since there are infinitely many poles of $f(z)$ in $D_{1}$, we will find a pole $z_{0}\left(\in D_{1}\right)$ of $f(z)$ such that $z_{0}-n_{1}\left(\in D_{1}\right)$ is a pole of $f(z)$ of multiplicity $k_{n_{1}}=(m-1)^{n_{1}-1} k_{1}$. And $z_{0}$ satisfies $z_{0}-\left(n_{1}+1\right) \notin D_{1}$, that is $\operatorname{Re}\left(z_{0}-\left(n_{1}+1\right)\right) \leq M$. By (2.1) and $m=p-q \geq 3$, we conclude that $z_{0}-\left(n_{1}+1\right)$ is a pole of $f(z)$ of multiplicity $k_{\left(n_{1}+1\right)}=(m-1) k_{n_{1}}=(m-1)^{n_{1}} k_{1}$. 
Substitute $z_{0}-\left(n_{1}+1\right)$ for $z$ in $(2.1)$ to obtain

$$
\begin{aligned}
f\left(z_{0}-\right. & \left.n_{1}\right) f\left(z_{0}-\left(n_{1}+1\right)\right)+f\left(z_{0}-n_{1}\right) f\left(z_{0}-\left(n_{1}+2\right)\right) \\
& +f^{2}\left(z_{0}-\left(n_{1}+1\right)\right)+f\left(z_{0}-\left(n_{1}+1\right)\right) f\left(z_{0}-\left(n_{1}+2\right)\right) \\
= & \frac{A_{0}\left(z_{0}-\left(n_{1}+1\right)\right)+\cdots+A_{p}\left(z_{0}-\left(n_{1}+1\right)\right) f^{p}\left(z_{0}-\left(n_{1}+1\right)\right)}{B_{0}\left(z_{0}-\left(n_{1}+1\right)\right)+\cdots+B_{q}\left(z_{0}-\left(n_{1}+1\right)\right) f^{q}\left(z_{0}-\left(n_{1}+1\right)\right)} .
\end{aligned}
$$

We see that the right-hand side of $(2.13)$ has a pole of multiplicity at least $p k_{\left(n_{1}+1\right)}-A-q k_{\left(n_{1}+1\right)}=m k_{\left(n_{1}+1\right)}-A$ at $z_{0}-\left(n_{1}+1\right)$. Without loss of generality, suppose that the right-hand side of (2.13) has a pole of multiplicity $m k_{\left(n_{1}+1\right)}-A$ at $z_{0}-\left(n_{1}+1\right)$.

In the left-hand side of (2.13), we see that $f^{2}(z)$ has a pole of multiplicity $2 k_{\left(n_{1}+1\right)}$ at $z_{0}-\left(n_{1}+1\right), \quad f(z+1) f(z)$ has a pole of multiplicity $k_{n_{1}}+k_{\left(n_{1}+1\right)}\left(<2 k_{\left(n_{1}+1\right)}\right) \quad$ at $\quad z_{0}-\left(n_{1}+1\right)$. By $m \geq 3$, when $n_{1}>$ $\max \left\{\frac{\log (A+1)-\log (m-2) k_{1}}{\log (m-1)}, 1\right\}$, we have $(m-2) k_{\left(n_{1}+1\right)}=(m-2)(m-1)^{n_{1}} k_{1}$ $>A+1>A$. Thus $m k_{\left(n_{1}+1\right)}-A>2 k_{\left(n_{1}+1\right)}$.

Hence, by (2.13), we conclude that $z_{0}-\left(n_{1}+2\right)$ is a pole of $f(z)$ of multiplicity $k_{\left(n_{1}+2\right)}=m k_{\left(n_{1}+1\right)}-A-k_{\left(n_{1}+1\right)}=(m-1) k_{\left(n_{1}+1\right)}-A=(m-1)^{n_{1}+1} k_{1}-A$.

We proceed to follow the steps as above. We will find $z_{0}-\left(n_{1}+n_{2}\right)$ is a pole of $f(z)$ of multiplicity $k_{\left(n_{1}+n_{2}\right)}=(m-1)^{n_{1}+n_{2}-1} k_{1}-A\left[(m-1)^{n_{2}-2}+\cdots+\right.$ $(m-1)+1]$ such that $\operatorname{Re}\left(z_{0}-\left(n_{1}+n_{2}\right)\right)<-M$, that is $z_{0}-\left(n_{1}+n_{2}\right) \in D_{2}$.

Then

Set $\quad k:=k_{\left(n_{1}+n_{2}\right)}=(m-1)^{n_{1}+n_{2}-1} k_{1}-A\left[(m-1)^{n_{2}-2}+\cdots+(m-1)+1\right]$.

$$
k=\frac{(m-1)^{n_{2}-1}}{m-2}\left[(m-2)(m-1)^{n_{1}} k_{1}-A\right]+\frac{A}{m-2} .
$$

When $\quad n_{2} \geq 2 \quad$ and $\quad n_{1}>\max \left\{\frac{\log (A+1)-\log (m-2) k_{1}}{\log (m-1)}, 1\right\}, \quad$ we have $(m-2)(m-1)^{n_{1}} k_{1}>A+1$, that is $(m-2)(m-1)^{n_{1}} k_{1}-A>1$. Hence we see that

$$
k=\frac{(m-1)^{n_{2}-1}}{m-2}\left[(m-2)(m-1)^{n_{1}} k_{1}-A\right]+\frac{A}{m-2} \geq 1 .
$$
$k \geq 1$.

Set $z_{1}:=z_{0}-\left(n_{1}+n_{2}\right)$. Then $z_{1}\left(\in D_{2}\right)$ is a pole of $f(z)$ of multiplicity obtain,

Applying the same reasoning that $f(z)$ has infinitely many poles in $D_{2}$, we

$$
n(r, f) \geq K(m-1)^{r}
$$

holds for all $r \geq r_{0}$. The fact that $r_{0}$ and $K$ both depend on $\left|z_{1}\right|$ is not a problem, since $z_{1}$ is fixed by $z_{0}$.

Furthermore, by (1.4), we have that

$$
\log \log M(r, f) \geq r \log \frac{m}{2}+\log K .
$$


Thus, by (2.14) we get

$$
\sigma_{2}(f)=\varlimsup_{r \rightarrow \infty} \frac{\log \log \log M(r, f)}{\log r} \geq \varlimsup_{r \rightarrow \infty} \frac{\log r+\log \left(\log \frac{m}{2}+\frac{\log K}{r}\right)}{\log r}=1 .
$$

And by (1.5), we have that

$$
\log n(r, f) \geq r \log (m-1)+\log K .
$$

Thus, by (2.15) we get

$$
\sigma_{2}(f)=\varlimsup_{r \rightarrow \infty} \frac{\log \log T(r, f)}{\log r} \geq \varlimsup_{r \rightarrow \infty} \frac{\log \log n(r, f)}{\log r} \geq 1 .
$$

Thus, Theorem 1.1 is proved.

\section{Proof of Theorem 1.2}

We need the following lemmas to prove Theorem 1.2.

Lemma 3.1 (see $[10,19])$. Let $f$ be a transcendental meromorphic solution of finite order $\sigma$ of the difference equation

$$
P(z, f)=0,
$$

where $P(z, f)$ is a difference polynomial in $f(z)$ and its shifts. If $P(z, a) \not \equiv 0$ for a slowly moving target function a, i.e. $T(r, a)=S(r, f)$, then

$$
m\left(r, \frac{1}{f-a}\right)=O\left(r^{\sigma-1+\varepsilon}\right)+S(r, f)
$$

outside of a possible exceptional set of finite logarithmic measure.

Lemma 3.2 (see [19]). Let $f$ be a transcendental meromorphic solution of finite order $\sigma$ of a difference equation of the form

$$
H(z, f) P(z, f)=Q(z, f),
$$

where $H(z, f)$ is a difference product of total degree $n$ in $f(z)$ and its shifts, and where $P(z, f), Q(z, f)$ are difference polynomials such that the total degree $\operatorname{deg} Q(z, f) \leq n$. If $H(z, f)$ contains just one term of maximal total degree, then for each $\varepsilon>0$,

$$
m(r, P(z, f))=O\left(r^{\sigma-1+\varepsilon}\right)+S(r, f)
$$

possibly outside of an exceptional set of finite logarithmic measure.

Lemma 3.3 (Valiron-Mohon'ko)(see [18]). Let $f(z)$ be a meromorphic function. Then for all irreducible rational function in $f$,

$$
R(z, f(z))=\frac{a_{0}(z)+a_{1}(z) f(z)+\cdots+a_{m}(z) f(z)^{m}}{b_{0}(z)+b_{1}(z) f(z)+\cdots+b_{n}(z) f(z)^{n}}
$$


with meromorphic coefficients $a_{i}(z)(i=0,1, \ldots, m), b_{j}(z)(j=0,1, \ldots, n)$, the characteristic function of $R(z, f(z))$ satisfies

$$
T(r, R(z, f(z)))=d T(r, f)+O(\Psi(r)),
$$

where $d=\operatorname{deg}_{f} R=\max \{m, n\}$ and $\Psi(r)=\max _{i, j}\left\{T\left(r, a_{i}\right), T\left(r, b_{j}\right)\right\}$.

In the remark of $[13$, p. 15], it is pointed out that the following Lemma 3.4 holds.

LEMMA 3.4. Let $f$ be a nonconstant finite order meromorphic function. Then

$$
N(r+1, f)=N(r, f)+S(r, f), \quad T(r+1, f)=T(r, f)+S(r, f)
$$

outside of a possible exceptional set of finite logarithmic measure.

Remark 2.1. In [8], Chiang and Feng proved that if $f$ is a meromorphic function with exponent of convergence of poles $\lambda\left(\frac{1}{f}\right)=\lambda<\infty, \eta \neq 0$ be fixed,
then for each $\varepsilon>0$,

$$
N(r, f(z+\eta))=N(r, f)+O\left(r^{\lambda-1+\varepsilon}\right)+O(\log r) .
$$

Lemma 3.5 (see [22]). Let $f_{j}(z)(j=1, \ldots, n)(n \geq 2)$ be meromorphic functions, $g_{j}(z)(j=1, \ldots, n)$ be entire functions, and satisfy

(i) $\sum_{j=1}^{n} f_{j}(z) e^{g_{j}(z)} \equiv 0$;

(ii) when $1 \leq j<k \leq n, g_{j}(z)-g_{k}(z)$ is not a constant;

(iii) when $1 \leq j \leq n, 1 \leq h<k \leq n, T\left(r, f_{j}\right)=o\left(T\left(r, e^{g_{h}-g_{k}}\right)\right),(r \rightarrow \infty, r \notin E)$, where $E \subset(1, \infty)$ is of finite linear measure or finite logarithmic measure. Then $f_{j}(z) \equiv 0(j=1, \ldots, n)$.

Lemma 3.6 (see [9]). Let $g:(0,+\infty) \rightarrow R, \quad h:(0,+\infty) \rightarrow R$ be nondecreasing functions. If (i) $g(r) \leq h(r)$ outside of an exceptional set of finite linear measure, or (ii) $g(r) \leq h(r), r \notin H \cup(0,1]$, where $H \subset(1, \infty)$ is a set of finite logarithmic measure, then for any $\alpha>1$, there exists $r_{0}>0$ such that $g(r) \leq h(\alpha r)$ for all $r>r_{0}$.

LEMma 3.7 (see [3]). Let $f(z)$ be a transcendental meromorphic function with $\sigma(f)<1$, and let $g_{1}(z)$ and $g_{2}(z)(\not \equiv 0)$ be polynomials, $c_{1}, c_{2}\left(\neq c_{1}\right)$ be constants. Then

$$
h(z)=g_{2}(z) f\left(z+c_{2}\right)+g_{1}(z) f\left(z+c_{1}\right)
$$

is transcendental.

Proof of Theorem 1.2. Suppose that $f(z)$ is a finite order transcendental meromorphic solution of (1.3). 
1) If $p=q=0$, then (1.3) can be rewritten as

$$
(f(z+1)+f(z))(f(z)+f(z-1))=\frac{a_{0}(z)}{b_{0}(z)} .
$$

Suppose that $a_{0}(z) \equiv 0$. Then we have that

$$
(f(z+1)+f(z))(f(z)+f(z-1))=0 .
$$

Thus, we have $f(z+1)+f(z) \equiv 0$ or $f(z)+f(z-1) \equiv 0$.

If $f(z+1)+f(z) \equiv 0$, then $f(z+1) \equiv-f(z)$, that is $f(z+2) \equiv f(z)$. Hence $f(z)$ is a periodic function. Therefore $\sigma(f) \geq 1$.

If $f(z)+f(z-1) \equiv 0$, by the same reasoning as above, we know $f(z)$ is a periodic function. Therefore $\sigma(f) \geq 1$.

Suppose that $a_{0}(z) \not \equiv 0$. Set $R(z)=\frac{a_{0}(z)}{b_{0}(z)}$. Then $R(z)$ has only finite many poles and zeros. Thus, there exists a real number $M>0$ such that all poles and zeros of $R(z)$ in $D=\{z:|z| \leq M\}$.

Contrary to the assertion $\sigma(f) \geq 1$, we suppose that $\sigma(f)<1$. Set $y(z)=$ $f(z)+f(z-1)$. By Lemma 3.7 and $\sigma(f)<1$, we conclude that $y(z)=f(z)+$ $f(z-1)$ is a transcendental meromorphic function. And (1.3) becomes the following difference equation

$$
y(z+1) y(z)=R(z) .
$$

And we have $\sigma(y) \leq \sigma(f)<1$. From (3.2), we know that $y(z+1)^{2}=$ $\frac{y(z+1)}{y(z)} R(z)$. By [8, Theorems 2.1, 2.2, Corollary 2.6], we obtain

$$
2 T(r, y(z))=T\left(r, \frac{y(z+1)}{y(z)}\right)+O(\log r)=N\left(r, \frac{1}{y(z)}\right)+N(r, y(z))+S(r, y) .
$$

So $y(z)$ must have infinitely many poles and zeros.

Set

$$
\begin{array}{ll}
D_{1}=\left\{z: \operatorname{Re} z>M_{1}\right\} ; & D_{2}=\left\{z: \operatorname{Re} z<-M_{1}\right\} ; \\
D_{3}=\left\{z: \operatorname{Im} z>M_{1}\right\} ; & D_{4}=\left\{z: \operatorname{Im} z<-M_{1}\right\},
\end{array}
$$

where $M_{1}=M+1$. Since $y(z)$ has infinitely many zeros, we see that there exists at least one of $D_{j}(j=1,2,3,4)$, say $D_{1}$, such that $y(z)$ has infinitely many zeros in $D_{1}$. Suppose that a point $z_{0} \in D_{1}$ satisfies $y\left(z_{0}\right)=0$. By (3.2), we see that $y\left(z_{0}+1\right)=\infty$ and $z_{0}+1 \in D_{1}$. Substituting $z_{0}+1$ into (3.2) to obtain

$$
y\left(z_{0}+2\right) y\left(z_{0}+1\right)=R\left(z_{0}+1\right) .
$$

By (3.3) and $y\left(z_{0}+1\right)=\infty$, we conclude that $y\left(z_{0}+2\right)=0$, and $z_{0}+2 \in D_{1}$.

Similarly, $z_{0}+2 n\left(\in D_{1}\right)$ is a zero of $y(z)$. Thus, there is a sequence $z_{0}, z_{0}+2, \ldots, z_{0}+2 n, \ldots$, they are zeros of $y(z)$. Thus, we get $\lambda(y) \geq 1$. A contradiction. 
If $y(z)$ has infinitely many zeros in $D_{3}$ (or $D_{4}$ ), then we may use the same method as above.

If $y(z)$ has infinitely many zeros in $D_{2}$, and $z_{0} \in D_{2}$ satisfies $y\left(z_{0}\right)=0$, then we can consider the other form of (3.2)

$$
y(z) y(z-1)=R(z-1) \text {. }
$$

By $y\left(z_{0}\right)=0$ and $z_{0} \in D_{2}$, we get $y\left(z_{0}-1\right)=\infty$ and $z_{0}-1 \in D_{2}$. Substituting $z_{0}-1$ into (3.4) to obtain

$$
y\left(z_{0}-1\right) y\left(z_{0}-2\right)=R\left(z_{0}-1\right) .
$$

By (3.5) and $y\left(z_{0}-1\right)=\infty$, we conclude that $y\left(z_{0}-2\right)=0$, and $z_{0}-2 \in D_{2}$.

Similarly, $z_{0}-2 n\left(\in D_{2}\right)$ is a zero of $y(z)$. Thus, we conclude that $z_{0}, z_{0}-2, \ldots, z_{0}-2 n, \ldots$ are zeros of $y(z)$. Thus, we get $\lambda(y) \geq 1$. A contradiction.

2) We prove that $\lambda(f)=\sigma(f)$. Set

$$
\begin{aligned}
E(z, f(z)):= & Q(z, f(z))(f(z+1)+f(z))(f(z)+f(z-1)) \\
& -a_{0}(z)-\cdots-a_{p}(z) f^{p}(z)=0 .
\end{aligned}
$$

Thus, since $a_{0}(z) \not \equiv 0$, we see that

$$
E(z, 0)=-a_{0}(z) \not \equiv 0 .
$$

By Lemma 3.1, we have that

$$
m\left(r, \frac{1}{f}\right)=O\left(r^{\sigma(f)-1+\varepsilon}\right)+S(r, f)
$$

outside of a possible exceptional set of finite logarithmic measure. So that

$$
N\left(r, \frac{1}{f}\right)=T(r, f)+O\left(r^{\sigma(f)-1+\varepsilon}\right)+S(r, f)
$$

outside of a possible exceptional set of finite logarithmic measure.

Hence, by Lemma 3.6, we have $\lambda(f)=\sigma(f)$.

3) By (1.3), we have that

$$
Q(z, f(z))(f(z+1)+f(z))(f(z)+f(z-1))=P(z, f(z)) .
$$

Since $q \geq p, q \geq 1$ and $P(z, f(z)) \not \equiv 0$, by Lemma 3.2 and (3.6), we have that

$$
m(r,(f(z+1)+f(z))(f(z)+f(z-1)))=O\left(r^{\sigma(f)-1+\varepsilon}\right)+S(r, f)
$$

possibly outside of an exceptional set of finite logarithmic measure. From Lemma 3.3 and (1.3), we get

$$
T(r,(f(z+1)+f(z))(f(z)+f(z-1)))=q T(r, f)+S(r, f) .
$$

By (3.7) and (3.8), we get

$$
N(r,(f(z+1)+f(z))(f(z)+f(z-1)))=q T(r, f)+O\left(r^{\sigma(f)-1+\varepsilon}\right)+S(r, f)
$$


possibly outside of an exceptional set of finite logarithmic measure. By Lemma 3.4 , we have that

$$
\begin{aligned}
N(r, & (f(z+1)+f(z))(f(z)+f(z-1))) \\
& \leq 2 N(r, f(z))+N(r, f(z+1))+N(r, f(z-1)) \\
& \leq 2 N(r, f(z))+2 N(r+1, f(z))=4 N(r, f(z))+S(r, f)
\end{aligned}
$$

possibly outside of an exceptional set of finite logarithmic measure. By (3.9) and (3.10), we get

$$
q T(r, f)+O\left(r^{\sigma(f)-1+\varepsilon}\right)+S(r, f) \leq 4 N(r, f(z))
$$

possibly outside of an exceptional set of finite logarithmic measure. Hence by Lemma 3.6 and (3.11), we get $\lambda\left(\frac{1}{f}\right) \geq \sigma(f)$. Thus, we get $\lambda\left(\frac{1}{f}\right)=\sigma(f)$.

4) If $a_{0}(z) \not \equiv 0$, by 2), we get $\max \left\{\lambda(f), \lambda\left(\frac{1}{f}\right)\right\}=\lambda(f)=\sigma(f)$. $\sigma(f)$.

If $q \geq p, q \geq 1$ and $P(z, f(z)) \not \equiv 0$, we have $\max \left\{\lambda(f), \lambda\left(\frac{1}{f}\right)\right\}=\lambda\left(\frac{1}{f}\right)=$

Thus, we only consider $p-q=1,2$ and $a_{0}(z) \equiv 0$. By Corollary 1.1, we see that $\operatorname{deg}_{f}(P) \leq 4, \operatorname{deg}_{f}(Q) \leq 2$ and $\operatorname{deg}_{f}(P)-\operatorname{deg}_{f}(Q) \leq 2$. Therefore, (1.3) has the following six forms:

$$
(f(z+1)+f(z))(f(z)+f(z-1))=a_{1}(z) f(z),
$$

where $a_{1}(z) \not \equiv 0$.

$$
(f(z+1)+f(z))(f(z)+f(z-1))=a_{1}(z) f(z)+a_{2}(z) f^{2}(z),
$$

where $a_{2}(z) \not \equiv 0$.

$$
(f(z+1)+f(z))(f(z)+f(z-1))=\frac{a_{1}(z) f(z)+a_{2}(z) f^{2}(z)}{b_{0}(z)+b_{1}(z) f(z)},
$$

where $a_{2}(z) b_{1}(z) b_{0}(z) \not \equiv 0$.

$$
(f(z+1)+f(z))(f(z)+f(z-1))=\frac{a_{1}(z) f(z)+a_{2}(z) f^{2}(z)+a_{3}(z) f^{3}(z)}{b_{0}(z)+b_{1}(z) f(z)}
$$

where $a_{3}(z) b_{1}(z) b_{0}(z) \not \equiv 0$.

$$
(f(z+1)+f(z))(f(z)+f(z-1))=\frac{a_{1}(z) f(z)+a_{2}(z) f^{2}(z)+a_{3}(z) f^{3}(z)}{b_{0}(z)+b_{1}(z) f(z)+b_{2}(z) f^{2}(z)},
$$

where $a_{3}(z) b_{2}(z) b_{0}(z) \not \equiv 0$.

$$
\begin{aligned}
(f(z & +1)+f(z))(f(z)+f(z-1)) \\
& =\frac{a_{1}(z) f(z)+a_{2}(z) f^{2}(z)+a_{3}(z) f^{3}(z)+a_{4}(z) f^{4}(z)}{b_{0}(z)+b_{1}(z) f(z)+b_{2}(z) f^{2}(z)},
\end{aligned}
$$

where $a_{3}(z) b_{2}(z) b_{0}(z) \not \equiv 0$. 
Suppose that $\max \left\{\lambda(f), \lambda\left(\frac{1}{f}\right)\right\}<\sigma(f)$. By Hadamard factorization theorem, $f(z)$ can be rewritten as

$$
f(z)=h(z) e^{d z^{n}},
$$

where $d(\neq 0)$ is a constant, $n(\geq 1)$ is an integer, $h(z)$ is a nonzero meromorphic function satisfying

$$
\sigma(h)=\max \left\{\lambda(f), \lambda\left(\frac{1}{f}\right)\right\}<\sigma(f) .
$$

Firstly, we consider equation $(1.3)_{1}$.

Substituting $f(z)=h(z) e^{d z^{n}}$ into $(1.3)_{1}$. We get

$$
\left(h(z+1) e^{d(z+1)^{n}}+h(z) e^{d z^{n}}\right)\left(h(z) e^{d z^{n}}+h(z-1) e^{d(z-1)^{n}}\right)=a_{1}(z) h(z) e^{d z^{n}} .
$$

Thus, we have that

$$
\left(h(z+1) h_{1}(z)+h(z)\right)\left(h(z)+h(z-1) h_{-1}(z)\right) e^{2 d z^{n}}=a_{1}(z) h(z) e^{d z^{n}},
$$

where $\quad h_{1}(z)=e^{d n z^{n-1}+\cdots+d} \quad$ and $\quad h_{-1}(z)=e^{-d n z^{n-1}+\cdots+(-1)^{n} d}$. Set $H(z)=$ $\left(h(z+1) h_{1}(z)+h(z)\right)\left(h(z)+h(z-1) h_{-1}(z)\right)$. By (3.12), we get

$$
H(z) e^{2 d z^{n}}-a_{1}(z) h(z) e^{d z^{n}} \equiv 0 .
$$

From Lemma 3.5 and (3.13), we have that $a_{1}(z) \equiv 0$. A contradiction. Hence we conclude that $\max \left\{\lambda(f), \lambda\left(\frac{1}{f}\right)\right\}=\sigma(f)$.

Similarly, we have $\max \left\{\lambda(f), \lambda\left(\frac{1}{f}\right)\right\}=\sigma(f)$ to equation $(1.3)_{2}$ except $(f(z+1)+f(z))(f(z)+f(z-1))=a_{2}(z) f^{2}(z)$.

Secondly, we consider equation $(1.3)_{3}$.

Substituting $f(z)=h(z) e^{d z^{n}}$ into $(1.3)_{3}$. We get

$$
\begin{aligned}
\left(h(z+1) e^{d(z+1)^{n}}+h(z) e^{d z^{n}}\right)\left(h(z) e^{d z^{n}}+h(z-1) e^{d(z-1)^{n}}\right) \\
=\frac{a_{1}(z) h(z) e^{d z^{n}}+a_{2}(z) h^{2}(z) e^{2 d z^{n}}}{b_{0}(z)+b_{1}(z) h(z) e^{d z^{n}}} .
\end{aligned}
$$

That is

$$
\begin{aligned}
\left(b_{0}(z)\right. & \left.+b_{1}(z) h(z) e^{d z^{n}}\right)\left(h(z+1) h_{1}(z)+h(z)\right)\left(h(z)+h(z-1) h_{-1}(z)\right) e^{2 d z^{n}} \\
= & a_{1}(z) h(z) e^{d z^{n}}+a_{2}(z) h^{2}(z) e^{2 d z^{n}}
\end{aligned}
$$

where $\quad h_{1}(z)=e^{d n z^{n-1}+\cdots+d} \quad$ and $\quad h_{-1}(z)=e^{-d n z^{n-1}+\cdots+(-1)^{n} d}$. Set $\quad H(z)=$ $\left(h(z+1) h_{1}(z)+h(z)\right)\left(h(z)+h(z-1) h_{-1}(z)\right)$. By (3.14), we get

$$
b_{1}(z) h(z) H(z) e^{3 d z^{n}}+\left[b_{0}(z) H(z)-a_{2}(z) h^{2}(z)\right] e^{2 d z^{n}}-a_{1}(z) h(z) e^{d z^{n}} \equiv 0 .
$$


From Lemma 3.5 and (3.15), we have that

$$
a_{1}(z) \equiv b_{0}(z) H(z)-a_{2}(z) h^{2}(z) \equiv b_{1}(z) h(z) H(z) \equiv 0 .
$$

Since $\quad b_{1}(z) h(z) \not \equiv 0$, we get $H(z) \equiv 0$. Thus $\quad b_{0}(z) H(z)-a_{2}(z) h^{2}(z) \equiv$ $-a_{2}(z) h^{2}(z) \equiv 0$. Moreover, $a_{2}(z) \equiv 0$. A contradiction.

Hence we conclude that $\max \left\{\lambda(f), \lambda\left(\frac{1}{f}\right)\right\}=\sigma(f)$.

Thirdly, we consider equation $(1.3)_{4}$.

Substituting $f(z)=h(z) e^{d z^{n}}$ into $(1.3)_{4}$. We get

$$
\begin{aligned}
\left(h(z+1) e^{d(z+1)^{n}}+h(z) e^{d z^{n}}\right)\left(h(z) e^{d z^{n}}+h(z-1) e^{d(z-1)^{n}}\right) & \\
= & \frac{a_{1}(z) h(z) e^{d z^{n}}+a_{2}(z) h^{2}(z) e^{2 d z^{n}}+a_{3}(z) h^{3}(z) e^{3 d z^{n}}}{b_{0}(z)+b_{1}(z) h(z) e^{d z^{n}}} .
\end{aligned}
$$

By the same reasoning as above, we get

$$
\begin{aligned}
& {\left[b_{1}(z) h(z) H(z)-a_{3}(z) h^{3}(z)\right] e^{3 d z^{n}}+\left[b_{0}(z) H(z)-a_{2}(z) h^{2}(z)\right] e^{2 d z^{n}}} \\
& \quad-a_{1}(z) h(z) e^{d z^{n}} \equiv 0 .
\end{aligned}
$$

From Lemma 3.5 and (3.16), we have that

$$
a_{1}(z) \equiv b_{0}(z) H(z)-a_{2}(z) h^{2}(z) \equiv b_{1}(z) h(z) H(z)-a_{3}(z) h^{3}(z) \equiv 0 .
$$

Since $h(z) \not \equiv 0$, we get $a_{2}(z)=\frac{b_{0}(z) H(z)}{h^{2}(z)}$ and $a_{3}(z)=\frac{b_{1}(z) H(z)}{h^{2}(z)}$. Thus

$$
a_{1}(z) f(z)+a_{2}(z) f^{2}(z)+a_{3}(z) f^{3}(z)=\frac{H(z)}{h^{2}(z)}\left[b_{0}(z)+b_{1}(z) f(z)\right] f^{2}(z) .
$$

This is a contradiction since $P(z, f(z))$ and $Q(z, f(z))$ are relatively prime polynomials in $f$.

Hence we conclude that $\max \left\{\lambda(f), \lambda\left(\frac{1}{f}\right)\right\}=\sigma(f)$.

Finally, we consider equations $(1.3)_{5}$ and $(1.3)_{6}$.

Substituting $f(z)=h(z) e^{d z^{n}}$ into $(1.3)_{5}$ and $(1.3)_{6}$ respectively. By the same reasoning as above, we get

$$
\begin{gathered}
b_{2}(z) h^{2}(z) H(z) e^{4 d z^{n}}+\left[b_{1}(z) h(z) H(z)-a_{3}(z) h^{3}(z)\right] e^{3 d z^{n}} \\
+\left[b_{0}(z) H(z)-a_{2}(z) h^{2}(z)\right] e^{2 d z^{n}}-a_{1}(z) h(z) e^{d z^{n}} \equiv 0
\end{gathered}
$$

and

$$
\begin{gathered}
{\left[b_{2}(z) h^{2}(z) H(z)-a_{4}(z) h^{4}(z)\right] e^{4 d z^{n}}+\left[b_{1}(z) h(z) H(z)-a_{3}(z) h^{3}(z)\right] e^{3 d z^{n}}} \\
+\left[b_{0}(z) H(z)-a_{2}(z) h^{2}(z)\right] e^{2 d z^{n}}-a_{1}(z) h(z) e^{d z^{n}} \equiv 0,
\end{gathered}
$$

respectively.

Similarly as above, from Lemma 3.5 and (3.17), we have that $a_{3}(z) \equiv 0$. A contradiction. 
Hence $\max \left\{\lambda(f), \lambda\left(\frac{1}{f}\right)\right\}=\sigma(f)$.

And by Lemma 3.5 and (3.18), we get $a_{2}(z)=\frac{b_{0}(z) H(z)}{h^{2}(z)}, a_{3}(z)=\frac{b_{1}(z) H(z)}{h^{2}(z)}$ and $a_{4}(z)=\frac{b_{2}(z) H(z)}{h^{2}(z)}$. Thus

$$
\begin{aligned}
a_{1}(z) & f(z)+a_{2}(z) f^{2}(z)+a_{3}(z) f^{3}(z)+a_{4}(z) f^{4}(z) \\
= & \frac{H(z)}{h^{2}(z)}\left[b_{0}(z)+b_{1}(z) f(z)+b_{2}(z) f^{2}(z)\right] f^{2}(z) .
\end{aligned}
$$

This is a contradiction since $P(z, f(z))$ and $Q(z, f(z))$ are relatively prime polynomials in $f$.

Hence we conclude that $\max \left\{\lambda(f), \lambda\left(\frac{1}{f}\right)\right\}=\sigma(f)$.
Theorem 1.2 is proved.

\section{Proof of Theorem 1.3}

Suppose that $f(z)$ is a rational solution of (1.6). Then $f(z)$ can be written as

$$
f(z)=\frac{m(z)}{n(z)}=\sum_{j=1}^{k}\left(\frac{c_{j \lambda_{j}}}{\left(z-z_{j}\right)^{\lambda_{j}}}+\cdots+\frac{c_{j 1}}{\left(z-z_{j}\right)}\right)+d_{0}+d_{1} z+\cdots+d_{s} z^{s},
$$

where $z_{j}(j=1, \ldots, k)$ are poles of $f(z)$ with multiplicities $\lambda_{j}$ respectively, $c_{j \lambda_{j}}(\neq 0), \ldots, c_{j 1}(j=1, \ldots, k)$ and $d_{0}, \ldots, d_{s}$ are constants.

1) Suppose first that $p>q$ and $p-q$ is an even number. By (1.6) and (4.1), we have

$$
\left(\frac{m(z+1)}{n(z+1)}+\frac{m(z)}{n(z)}\right)\left(\frac{m(z)}{n(z)}+\frac{m(z-1)}{n(z-1)}\right)=\frac{P(z)}{Q(z)} .
$$

If $\operatorname{deg} m(z)=m<n=\operatorname{deg} n(z), \quad$ then $\quad \frac{m(z+1)}{n(z+1)} \rightarrow 0, \quad \frac{m(z)}{n(z)} \rightarrow 0 \quad$ and $\frac{m(z-1)}{n(z-1)} \rightarrow 0$ as $z \rightarrow \infty$. But $\frac{P(z)}{Q(z)} \rightarrow \infty$ as $z \rightarrow \infty$. Thus (4.2) is a contradiction. If $m=n$, then $\frac{m(z+1)}{n(z+1)} \rightarrow a, \frac{m(z)}{n(z)} \rightarrow a$ and $\frac{m(z-1)}{n(z-1)} \rightarrow a$ as $z \rightarrow \infty$, where $a$ is a nonzero constant. This is also a contradiction. So $m>n$. Thus we can assume that $d_{s} \neq 0(s \geq 1)$. Since for sufficiently large $z$,

$$
\left\{\begin{array}{l}
f(z)=d_{s} z^{s}(1+o(1)), \\
f(z+1)=d_{s} z^{s}(1+o(1)), \\
f(z-1)=d_{s} z^{s}(1+o(1)), \\
\frac{P(z)}{Q(z)}=A^{*} z^{p-q}(1+o(1)),
\end{array}\right.
$$


where $A^{*}(\neq 0)$ is some constant. By (1.6) and (4.3), we obtain

$$
4 d_{s}^{2} z^{2 s}(1+o(1))=A^{*} z^{p-q}(1+o(1))
$$

for sufficiently large $z$. So, by (4.4), we have that

$$
m-n=s=\frac{p-q}{2} .
$$

Now we suppose that $p=q$. Thus for sufficiently large $z$

$$
\frac{P(z)}{Q(z)}=A^{*}(1+o(1)),
$$

where $A^{*}(\neq 0)$ is some constant. If $m<n$, then using the same method as above, we get a contradiction. If $m \geq n$, then we may assume that $d_{s} \neq 0$ $(s \geq 0)$. Using the same method as above, we have that for sufficiently large $z$,

$$
4 d_{s}^{2} z^{2 s}(1+o(1))=A^{*}(1+o(1)) .
$$

Hence we have $m-n=0=\frac{p-q}{2}$.

2) Suppose that $p<q$ and $q-p$ is an even number. By (1.6) and (4.1), we also obtain (4.2).

If $\operatorname{deg} m(z)=m>n=\operatorname{deg} n(z), \quad$ then $\quad \frac{m(z+1)}{n(z+1)} \rightarrow \infty, \quad \frac{m(z)}{n(z)} \rightarrow \infty \quad$ and $\frac{m(z-1)}{n(z-1)} \rightarrow \infty$ as $z \rightarrow \infty$. But $\frac{P(z)}{Q(z)} \rightarrow 0$ as $z \rightarrow \infty$. Thus (4.2) is a contradiction. If $m=n$, then $\frac{m(z+1)}{n(z+1)} \rightarrow a, \frac{m(z)}{n(z)} \rightarrow a$ and $\frac{m(z-1)}{n(z-1)} \rightarrow a$ as $z \rightarrow \infty$, where $a$ is a nonzero constant. This is also a contradiction. So $m<n$. By (4.2) we obtain

$$
\begin{aligned}
Q(z)[ & m(z+1) m(z) n(z-1) n(z)+m(z+1) m(z-1) n^{2}(z) \\
& \left.+m^{2}(z) n(z+1) n(z-1)+m(z) m(z-1) n(z+1) n(z)\right] \\
= & P(z) n^{2}(z) n(z+1) n(z-1) .
\end{aligned}
$$

Set

$$
\left\{\begin{array}{l}
m(z)=a_{m} z^{m}+\cdots \\
n(z)=b_{n} z^{n}+\cdots \\
P(z)=d_{p} z^{p}+\cdots \\
Q(z)=l_{q} z^{q}+\cdots
\end{array}\right.
$$

where $n \geq 1, m \geq 0$ and $q \geq 1, p \geq 0$. By (4.5) and (4.6), we obtain

$$
4 l_{q} a_{m}^{2} b_{n}^{2} z^{q+2 m+2 n}+\cdots=d_{p} b_{n}^{4} z^{p+4 n}+\cdots .
$$

By (4.7), we see that $n-m=\frac{q-p}{2}$ and $\frac{a_{m}^{2}}{b_{n}^{2}}=\frac{d_{p}}{4 l_{q}}$. 
3) If $p>q$, then $|p-q|=p-q$ is an odd number. Suppose that equation (1.6) has a rational solution $f(z)=\frac{m(z)}{n(z)}$. From the proof in 1), we see that if $p>q$, then $m-n=\frac{p-q}{2}$. This contradicts our supposition that $p-q$ is an odd number. So, (1.6) has no rational solution.

If $p<q$, then $|p-q|=q-p$ is an odd number. Using the same method as above, we conclude that (1.6) has no rational solution.

Thus, the Theorem 1.3 is proved.

\section{REFERENCES}

[1] M. Ablowitz, R. G. Halburd and B. Herbst, On the extension of Painlevé property to difference equations, Nonlinearity 13 (2000), 889-905.

[ 2 ] W. Bergweiler and J. K. Langley, Zeros of differences of meromorphic functions, Math. Proc. Cambridge Philos. Soc. 142 (2007), 133-147.

[ 3 ] Z. X. Chen, On growth, zeros and poles of meromorphic solutions of linear and nonlinear difference equations, Sci. China Math. 54 (2011), 2123-2133.

[4] Z. X. Chen And K. H. Shon, On zeros and fixed points of differences of meromorphic functions, J. Math. Anal. Appl. 344 (2008), 373-383.

[5] Z. X. CHEN, Value distribution of meromorphic solutions of certain difference Painlevé equations, J. Math. Anal. Appl. 364 (2010), 556-566.

[6] Z. X. ChEN, Growth and zeros of meromorphic solution of some linear difference equations, J. Math. Anal. Appl. 373 (2011), 235-241.

[7] Z. X. CHEN, On properties of meromorphic solutions for some difference equations, Kodai Math. J. 34 (2011), 244-256.

[8] Y. M. Chiang and S. J. Feng, On the Nevanlinna characteristic of $f(z+\eta)$ and difference equations in the complex plane, Ramanujan J. 16 (2008), 105-129.

[9] G. Gundersen, Finite order solutions of second order linear differential equations, Trans. Amer. Math. Soc. 305 (1988), 415-429.

[10] R. G. Halburd and R. Korhonen, Difference analogue of the lemma on the logarithmic derivative with applications to difference equations, J. Math. Anal. Appl. 314 (2006), 477-487.

[11] R. G. Halburd and R. Korhonen, Nevanlinna theory for the difference operator, Ann. Acad. Sci. Fenn. Math. 31 (2006), 463-478.

[12] R. G. Halburd and R. Korhonen, Existence of finite-order meromorphic solutions as a detector of integrability in difference equations, Phys. D 218 (2006), 191-203.

[13] R. G. Halburd and R. Korhonen, Meromorphic solution of difference equations, integrability and the discrete Painlevé equations, J. Phys. A 40 (2007), 1-38.

[14] R. G. Halburd, R. J. Korhonen And K. Tohge, Holomorphic curves with shift-invariant hyperplane preimages, arXiv: 0903.3236, 1-30.

[15] W. K. Hayman, Meromorphic functions, Clarendon Press, Oxford, 1964.

[16] J. Heittokangas, R. Korhonen, I. Laine, J. Rieppo and K. Tohge, Complex difference equations of Malmquist type, Comput. Methods Funct. Theory 1 (2001), 27-39.

[17] J. Heittokangas, R. Korhonen, I. Laine, J. Rieppo and J. Zhang, Value sharing results for shifts of meromorphic functions, and sufficient conditions for periodicity, J. Math. Anal. Appl. 355 (2009), 352-363. 
[18] I. LAINE, Nevanlinna theory and complex differential equations, Walter de Gruyter, Berlin, 1993.

[19] I. LAINE AND C. C. YANG, Clunie theorems for difference and q-difference polynomials, J. Lond. Math. Soc. 76 (2007), 556-566.

[20] A. Ramani, B. Grammaticos, T. Tamizhmani and K. M. Tamizhmani, The road to the discrete analogue of the Painlevé propertiy: Nevanlinna meets singularity confinement, Comput. Math. Appl. 45 (2003), 1001-1012.

[21] C. C. YANG AND I. LAINE, On analogies between nonlinear difference and differential equations, Proc. Japan Acad. 86 (2010), 10-14.

[22] C. C. YANG AND H. X. YI, Uniqueness theory of meromorphic functions, Dordrecht: Kluwer Academic Publishers Group, 2003.

[23] L. YANG, Value distribution theory, Science Press, Beijing, 1993.

Chang-Wen Peng

School of Mathematics and Computer Sciences

GuiZhou Normal College

GUIYANG 550018

P. R. CHINA

E-mail: pengcw716@126.com

Zong-Xuan Chen

School of Mathematical Sciences

South China Normal University

GuANGZHOU 510631

P. R. CHINA

E-mail: chzx@vip.sina.com 